\title{
Developing an Intelligent Framework for Improving the Quality of Service in the Government Organizations in the Kingdom of Saudi Arabia
}

\author{
Abdulelah Abdallah AlGosaibi ${ }^{1}$, Abdul Rahaman Wahab Sait ${ }^{2}$, Abdulaziz Fahad AlOthman ${ }^{3}$, Shadan AlHamed ${ }^{4}$ \\ College of Computer Science and Information Technology, King Faisal University, Al-Ahsa, Kingdom of Saudi Arabia ${ }^{1}$ \\ Department of Documents and Archives, King Faisal University, Al-Ahsa, Kingdom of Saudi Arabia 2,3,4
}

\begin{abstract}
The Kingdom of Saudi Arabia is enhancing the services and applications in government organizations through the number of systems that generate a massive amount of data through Big Data technology. Recently, the Global Artificial Intelligent Summit 2020, Saudi Data and Artificial Intelligence Authority (SDAIA), NEOM have launched an Artificial Intelligence (AI) strategy that aligns with the Kingdom Vision 2030. AI opens a wide door for opportunities and new strategies that will narrow the gap in the skillset of individuals and promote research and innovation in the IT industry. Organizations lack advanced techniques to evaluate the performance of individuals and departments that supports improving the quality of service. The introduction of AI-based applications in the government and private sectors will facilitate decision-makers in tracking and optimizing the efficiency of departments and individuals. This research aims to develop an intelligent framework for government organizations to improve the quality of services rendered to customers and businesses. In addition, it highlights the importance of AI policies in archiving metadata. This paper presents a framework for an organization that contains Chatbot, Sentiment Analysis, and Key Performance Indicators to improve the services. A synthetic dataset is employed as a testbed to evaluate the performance of the framework. The outcome of this study shows that the proposed framework able to improve the performance of organizations. Using this proposed framework, organizations can build a mechanism for their workforce to retrieve meaningful information. Moreover, it provides significant features include efficient data extraction, data management, and AI-based security for effective document management.
\end{abstract}

Keywords-Key performance indicators; big data; hierarchical analysis; artificial intelligence; privacy policies; metadata; pattern generation

\section{INTRODUCTION}

The vision 2030 of the Kingdom of Saudi Arabia has initiated many plans and programs to enhance the performance of Education, Medicine, and other core sectors. Skill development and performance improvement are the two essential goals of vision 2030 [1]. In recent days, data produced widely from various resources. Artificial Intelligence (AI) and Big Data (BD) are the key players in transforming and empowering modern organizations process that improves decision-making, trend analysis, forecasts, etc. [2][3]. Valuable information from them by applying BD analytics to stored data. And, sophisticated AI algorithms are used to obtain insights into consumer behaviour in order to grasp current patterns and stand high among competitors [4]. BD monitors the performance of workers, design precise delivery models, predictive maintenance, etc. Few organizations use this technology to create a smart workplace and track their employees [5]. The use of metadata in global enterprises is vital in determining the success of a sustainable organization and in gathering insight. Metadata provides meta-information about communication that occurred between two or more individuals or departments. Identifying the invaluable management strategies that can minimize costs, assess how far a company advances and equate them to its organizational objectives [6][7]. This inspiration from top-level motivates organizations to enhance current functionally and lunch new advanced one. Therefore, there is a demand for intelligent data analytical tools for measuring the performance of each department and individual in Government Organization (GO). The availability of sophisticated tools and techniques provide an opportunity for decision-maker to make an effective decision that improves individual performance which leads to organization success [6][9][10]. Data relating to document flow in an organization would be a supporting and influencing factor for the 2030 vision and objective. It can be used to monitor the performance of administrative departments and employees. The data collection process will begin with the creation of correspondence in a particular department and collects data, including receiver information, degree of confidentiality, urgency, and automatically created data, such as the name of the sender, correspondence time, and data, and transmission information. Such data can be employed to provide a base for achieving an individual's efficiency. Recently, in the Global Summit 2020, the strategy for AI and data is announced by Saudi Data and Artificial Intelligence Authority (SDAIA). The plan supports individual to learn AI technology and develop their skills. Also, it assists organizations in applying AI to utilize the resources effectively. Furthermore, GOs are following the rules and policies of the government. Therefore, there is an exigency for GO to improve their functionalities in order to provide a better service to the public.

AI-driven Data Management System (DMS) can enable organizations to reduce data redundancy, prevent data losses, and prevent the recurrence of multiple input errors [11]. In the document management system, data analytics is one of the most critical functions of artificial intelligence. Adequate and 
comprehensive analysis of data will contribute to the growth of the enterprise. It is also essential to secure documents, susceptible ones, along with the management of records [12]. AI-powered DMS provides the highest levels of document protection by detecting confidential and personal information from documents. Data can be generated from various sources with advanced technology; however, this data needs to be analyzed and organized to gather useful information. The management and extraction of knowledge from documents will save a lot of time and effort. Documents can be clustered and analyzed by an artificial intelligence and Machine Learning (ML) framework [13]. It supports to understand the essence of a document and its relationship with other documents. Artificial intelligence technology may use optical character recognition to construct a document management system to interpret what is on a document to classify it accurately. Based on the categorization, it aids further in automating workflow processes. $\mathrm{BD}$ is one of the latest technologies and frameworks that can use real-time processing and analysis to maximize the value of substantial quantities of highly diverse data.

The introduction of BD has provided an opportunity for an organization to adopt multiple applications and services to enhance its performance. Recently, large quantities of data have been generated from various government outlets, such as the internet, cloud systems, and mobile devices [3][14]. Each $\mathrm{GO}$ is maintaining a large data corpus through BD and communicating with other organizations. In addition, employees and departments are availing of the services in the BD environment, and the size of data is increasing exponentially [3][15]. E-governance is the use of new communication and information technology to enhance organization performance, efficacy, and service delivery, and to encourage accountability [5][16][17][18]. Both BD and Egovernance offers decision-makers to make a better decision and improve the organization performance that leads to customer/client satisfaction. Most organizations' mission or objective facilitates the establishment of products and services. They have to procure resources, transform them into outputs, and deliver them to their intended users.

Both $\mathrm{AI}$ and $\mathrm{BD}$ provide an opportunity for firms to understand their customers. BD offers completely new levels of exploration of hidden outcomes. In the past, organizations were unable to analyze such large amounts of data, and now that the ability to do that would lead to unintended business benefits. The consistency of the details is one more added challenge. Some documents do not even comply with the minimum clarity bar. BD defines the high amount of structured and unstructured data that floods an organization regularly. The storage of $\mathrm{BD}$ is distributed over several computers to reduce the cost of implementation and maintenance. Moreover, it supports the search algorithm to search in a specific memory instead of a huge memory/dataset.

Sentiment analysis utilizes Natural Language Processing (NLP) and ML in data sources to perceive and identify emotions [19]. It is also used in business to identify social data sentiment, assess the company's reputation, and understand clients. It recognizes and categorizes the opinion expressed in the text, such as news stories, content on social media, ratings, etc. Similar to many NLP techniques, it needs to be able to deal with the language complexities [20]. Organizations must understand people's emotions as clients share their thoughts and feelings more freely than ever before. The automated identification of the type of correspondence in the document centre assists a department or individual to respond in time.

Chatbots are small programs that assist users dynamically based on a set of predefined conditions, and events [19][20]. Chatbots are used for different purposes in interactive systems, including customer service, request routing, or information collection. While some chatbot implementations use comprehensive word classification processes, natural language processors, and advanced AI, others use common phrases obtained from a related library or database to search for general keywords and produce responses.

The role of KPIs within the organizational framework is to provide customizable and readily accessible measurement to users/clients to improve the quality and effectiveness of their operations [6][7]. The significance of using KPIs relies on how effectively they support better to understand the factors for an organization's success. For instance, a government company such as a university or a hospital would have different fiscal KPIs than a publicly traded corporation [7]. Each KPI should represent the organization's mission and objectives. A common mistake in creating KPIs is that a declaration such as "Improve productivity of a department" as a KPI is too general. It needs to be precise and measurable, like "Improving the individual performance of a specific department in a specific period" in order to be successful [6]. KPIs are crucial to tracking progress in publishing and reporting. Report formats should be tailored to position and purpose so that managers see a summary view, whereas department heads have much more comprehensive measurements [21]. The combination of dashboards, scorecards, informative reports, and ad-hoc research selfservice tools should be considered. The KPI results allow decision-makers to determine where the emphasis should be placed to enhance the department/organization's performance [6]. In addition, Analytics serve to build a strategic plan based on the knowledge available. The creation of standardized realtime dashboards requires an investment of time and much more time to use the data effectively. Individuals will be notified of issues based on data analysis, identify potential solutions, and receive ideas for new opportunities.

In the analytical process, ML will assist, clearly identify odd trends in procedures, and send suggestions about what to do next; this process can provide useful AI insights. By developing consistent operating metrics and evaluating efficiency, businesses benefit from the use of what is necessary for being competitive on the market and provide adequate services to the customers. Key Performance Indicator (KPI) refers to a collection of quantifiable metrics used to assess the overall long-term performance of an organization. It supports to evaluate the strategic, financial, and operational achievements of a business, especially relative to those of other firms in the same industry [6][21]. Fig. 1 shows the analytical framework of the proposed research. Metadata is the primary source for this research. BD provides metadata to generate meaningful information through AI applications and KPI. 


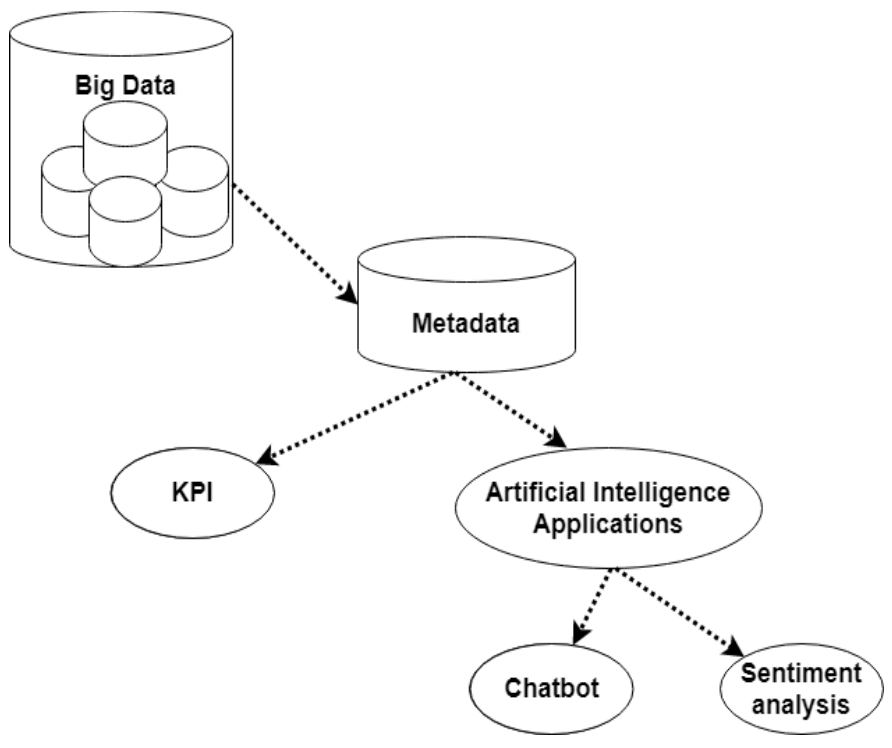

Fig. 1. Proposed Techniques for Analyzing Metadata.

The study aims to develop a framework that AI and BD concerning organization needs, which include Sentiment Analysis, Automated ChatBot, and analytical techniques for government organizations in the Kingdom of Saudi Arabia. The proposed framework support organizations to improve the quality of service rendered to the customers. It assesses organizations to achieve the objectives of vision 2030 of the Kingdom of Saudi Arabia.

Hierarchical analysis technique analyses the organization tree structure and support decision-makers/administrators to measure the performance of their department/employee. Moreover, the study discusses privacy and policy issues related to $\mathrm{AI}$ and $\mathrm{BD}$. BD, E-Governance, and $\mathrm{AI}$ are the elements of a modern organization framework.

The structure of the study is organized as follows: Section 2 discusses the related works of BD, AI, and KPI in government organizations. Section 3 provides the methodology to develop KPI for BD applications. Section 4 describes the outcomes of the proposed framework, and Section 5 discusses the achievements of the proposed framework. Finally, Section 6 concludes the study with its future direction.

\section{RELATED WORKS}

BD analytics is stated as a method to analyze data and discover hidden trends [22]. The exponential growth of data and its analysis using different mining methods leads to a better understanding of workforce in an enterprise. E-Governance is a decision-making and implementation process that ensures a country's resources are applicable, accessible, and driven [18]. The BD framework provides an opportunity for organizations to combine heterogeneous data. Moreover, it offers individuals and corporations a global chance of combining a wide range of public databases, fraud-detection, and tax compliance activities to enhance services and apps. The effect of BD on these egovernment applications supports to promote better governance [17].
In [2], the authors discussed the opportunities of implementing $\mathrm{AI}$ in the workplace to support administrators in making effective decisions. They presented an empirical study that evaluated and analyzed to what extent enterprises employ analytical tools for predicting business outcomes. The outcome of the study shows that an AI-based workplace supports decision-makers in complex situations.

In [3], the authors identified the challenges related to the implementation of AI-based applications to make decisions. They offered several research propositions for Information system researchers. They discussed the issues associated with the deployment of AI and replacing the manual decisionmaking process. In [5], a case study related to E-Governance is discussed. The authors analyzed the existing E-Governance system in Odisha, India. The findings of the case study that the deployment of data analytical tools in the workplace have improved the work environment.

AI applications such as Chatbot and Sentiment Analysis supports the customers of an organization by providing responses and required services. Organizations are effectively and efficiently managing documents through AI document analysis, data extraction, ML for content management, and Information security. BD is regarded as a reliable and useful source that can be analyzed with AI applications [14]. The research forecasts and makes decisions that may increase the reputation of the business. $\mathrm{BD}$ and $\mathrm{AI}$ are two concepts that are commonly used when describing the company's future [5][14][15]. The opportunity to implement them in various aspects of the business has captured the imagination of many, specifically, how AI can reduce the resources and provide a more secured work environment. BD and AI could adapt business processes and decisions to meet individual needs and preferences, enhancing process and decision efficiency [5][14]. Although wide advantages of $\mathrm{BD}$ and $\mathrm{AI}$ can be anticipated, several risks involve some policy considerations [11]. Governments will potentially establish general guidelines or legislation related to the use of $\mathrm{BD}$ and the application of $\mathrm{AI}$, and the enterprise should be prepared to implement them.

In [7], the authors addressed the importance of KPI in improving the quality of services rendered to the customer. Different types of KPIs were discussed in this study. In [8], authors developed the performance indicators to operate and maintain an enterprise. The presence of a wide variety of analytics tools assists enterprises to organize their departments and employees in a better way. However, the absence of an efficient set of KPIs leads to issues such as less productivity, unorganized departmental activities, and low performance. Therefore, a demand for KPI which reveals hidden pattern via intelligent and hierarchical analysis of organizational tree structure. KPIs are essential to effective management by identifying underperforming aspects of the business as well as that demand increased resources [8]. It will report events that may signify problems that arise internally within the organization's operations or potential threats resulting from external events may provide management with rich information to improve the organization's strategies [6].

In [20], the applications of AI-based customer assistance in the online shopping portal are discussed. Developers follow 
two approaches to develop an AI Chatbot to identify the user's needs and respond with relevant responses. One of the development approaches is that the AI robot must respond to a user query with a restricted set of guidelines and internal structuring. A bag of words can be generated in such a way to map frequently used questions with appropriate solutions [23][24][25]. For instance, an automated banking bot asks the caller a series of questions to know what he or she needs. The AI bot will either repeat the instruction or pass the call to a human banking assistant if the caller gives an order that is out of reach. The second way to communicate with an AI chatbot is to know what a user is looking for and to deliver real-time answers based on progressive conversations or enhanced learning [2]. Although due to its complexity, this mechanism is still emerging, some apps such as Amazon's Alexa, Google Assistant, and Facebook AI-Chatbot are on the road to dynamic responses based on human behavioral and preferential traits [12][26]. Self-learning is a technique to understand the human mind and obtain enough knowledge to generate persuasive responses [27][28]. It is called Intelligent Chatbots or general AI chatbots. A general-purpose AI chatbot can understand language and continue learning based on the inputs and analyzing sentiments [29][30][31].

Based on the analysis of the related works, the study intends to develop a framework that applies $\mathrm{AI}$ and $\mathrm{BD}$ to enhance the processes in the government organizations as per the Vision 2030 of the Kingdom of Saudi Arabia. The proposed framework aims to assist end-users in deriving value from the data corpus. The implementation of an AI-based framework provides tools and techniques for decision-makers to analyze and monitor the workforce. Moreover, it secures the document by applying AI policies and report the status to the administration.

\section{MAterials AND Methods}

Synthetic metadata is developed based on the assumptions on the functionality and structure of the organizations in the Kingdom of Saudi Arabia. The following part of this section introduces the AI-based application. Table I presents the notations used in the proposed algorithms for Sentiment Analysis and KPI. The notations are used as a parameter in the following algorithm. It indicates the input required for the proposed technique to produce an output. For context, Sentiment analysis requires metadata to generate the type of correspondence. Similarly, the following notations are indicating an input or output of the proposed methods.

\section{A. ChatBot}

The primary sources for this application are correspondences, history of correspondences, and details of employees and departments. The proposed AI chatbot uses a bag of words technique to identify the users and respond to them with a relevant response. Initially, metadata related to department and employee are extracted and refined and stored as Bag of Words. ChatBot receives input from the user query and parses it to derive tokens. The query tokens are passed into the Bag of words to find the existence of relevant tokens. And, based on the frequency of the tokens, the response will be returned to the user.

\section{B. Sentiment Analysis}

Sentiment analysis is based on the details of internal, incoming, and outgoing correspondences. The supervised learning-based Naive Bayes (NB) algorithm is employed to find a pattern from the metadata and predict the type of user correspondences. It supports users to complete correspondence in a limited time with interactive solutions. During the training phase, the labels are required to train the proposed technique. The labels are extracted from the metadata(D). It includes the type of correspondence, urgency level, and department level are used as a level. A conditional probability is generated using tokens and returned as an output with vocabulary. Fig. 2 provides the testing phase of the sentiment analysis. The testing phase able to predict the type of correspondence using the conditional probability of data. An NB classifier is used to classify the type of correspondence and helps to complete an urgent correspondence in a limited amount of time. The notations used in Fig. 2 were explained in the initial part of this section.

\section{Key Performance Indicators}

The organization's tree structure is classified into four levels: Root-level, Level - 2 department, Department, and Employees. The processes in the development of KPI and classification of Department and Employees are shown in Fig. 3. And, the initial processes are representing the identification of KPI to analyze each level individually and hierarchically. The performance of the proposed KPI is measured by computing the total correspondence for Root and Level-1 departments, respectively.

TABLE I. NOTATION AND MEANING

\begin{tabular}{|l|l|l|l|}
\hline Notation & Meaning & Notation & Meaning \\
\hline $\mathrm{D}$ & Metadata & $\mathrm{Cd}$ & $\begin{array}{l}\text { Classified } \\
\text { departments }\end{array}$ \\
\hline $\mathrm{d}$ & Department & $\mathrm{Ce}$ & Classified employees \\
\hline $\mathrm{W}$ & Vocabulary (Initial) & $\mathrm{e}$ & Employees \\
\hline $\mathrm{V}$ & $\begin{array}{l}\text { Vocabulary (Testing } \\
\text { phase) }\end{array}$ & $\mathrm{C}$ & Class \\
\hline $\mathrm{t}_{\mathrm{m}}$ & Term & & \\
\hline
\end{tabular}

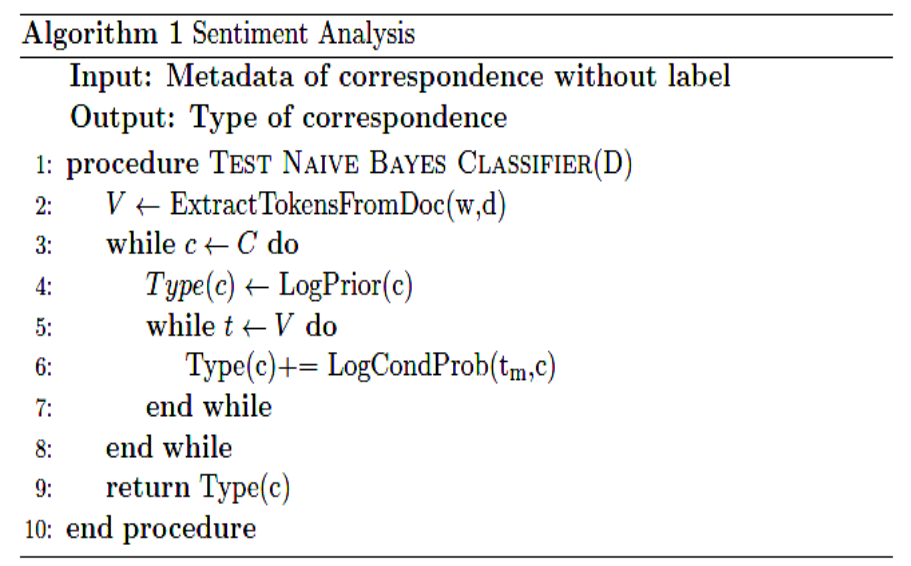

Fig. 2. Proposed Algorithm for Sentiment Analysis. 


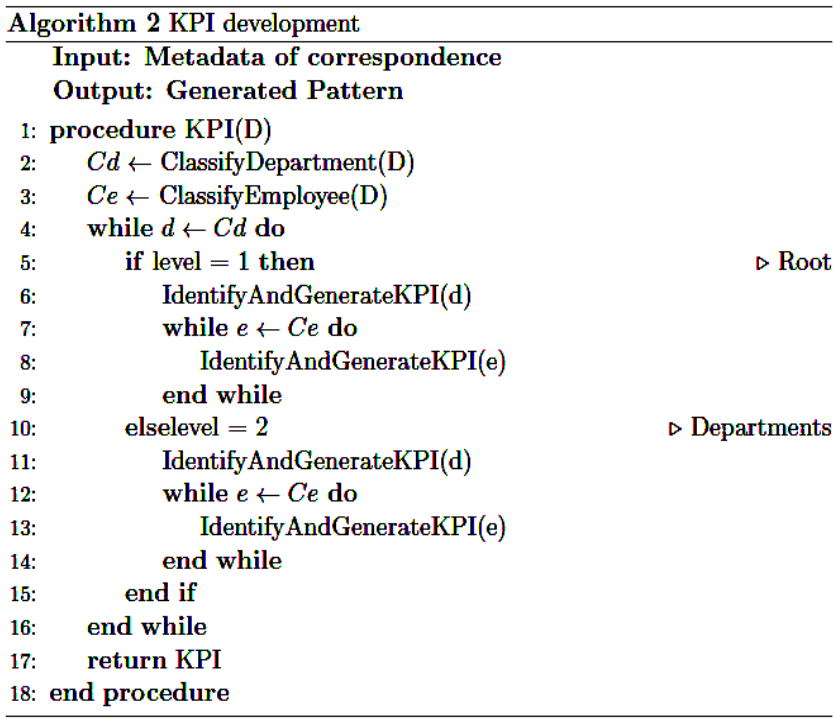

Fig. 3. Proposed Algorithm for KPI Development.

Fig. 4 illustrates the classified departments and employees in an organization. The Root / Senior management (level - 1) for entire departments in an enterprise. Level - 2 departments are in level -2 , which indicates a cluster of departments. Departments are in level -3 that represents single / multiple departments and finally, Employees are in level $-\mathrm{n}$ to mean an individual in an enterprise. Each employee may have multiple roles to represent their activities in different departments.

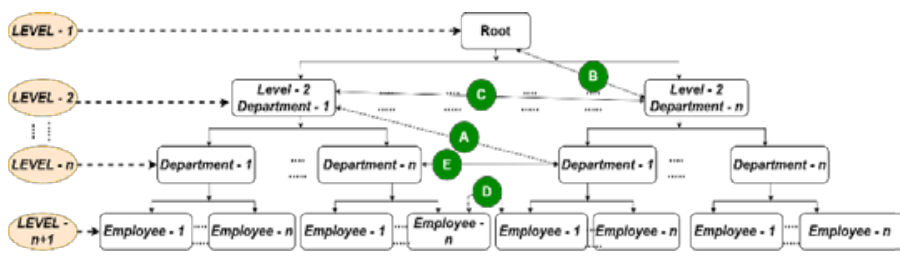

Fig. 4. Hierarchical Analysis in the Organization's Tree Structure.

Senior management has a management level as a subdepartment under level -1 . In the same way, each management and departments are classified with relevant sub-departments and employees. The Level -1 , senior management have access to all levels and privilege to have direct departments under it. As per the organization tree, Level -1 is a root (Senior management), Level -2 is the parent departments for the subsequent levels, Level - 3 is Department, and finally, Level $-\mathrm{n}$ is Employee.

A standard set of KPI is identified for all four levels (Root, Level-2 department, and Department, and Employee) to calculate the number of correspondences between two departments. In Fig. 4, the link " $\mathrm{B}$ " is used to show the transaction between Root and Level - 2 departments, i.e., level -1 and Level -2 . For instance, suppose the Root, Level -1 has sent a total of 17 correspondences to Level -2 department $-\mathrm{n}$ of Level -2 . The information about the interactions can be extracted using the proposed framework. The link "C" is used to represent the connection between Level - 2 departments in the same Level. For context, suppose a Level - 2 department 1 of Level -2 has sent a total of 16 correspondences to another Level - 2 department - $\mathrm{n}$ of Level -2 . These details can be extracted through the proposed framework. The links "A", " $\mathrm{D}$ ", and " $\mathrm{E}$ " is also used to interact with the different levels of the organization tree structure. The proposed framework provides an opportunity to extract specific correspondence between different levels as well as in the same Level.

\section{RESULTS}

In this section, the implementation of the proposed framework is discussed. Based on the functionality of the organizations, a dataset is developed similar to metadata about document transactions. The proposed KPI, Chatbot, and Sentiment analysis are implemented and tested with the synthetic dataset. The findings will not reflect the actual hierarchy of any organization.

\section{A. Chatbot}

The researchers succeeded in the initial attempt in developing a Chatbot application using metadata that provides an automated solution to the user query. Python 3.8.1 is used for the development of Chatbot. Metadata are extracted from the database and stored in Comma Separated Value (CSV) format. The following Fig. 5 shows the initial set of results in Chatbot development. When a user logged into the application, the automated Bot has used the username and retrieved the department id and his / her last correspondence history and the total number of correspondences. The date format is represented in the Hijri date format. The proposed Chatbot uses the metadata of an organization correspondence history and correspondence forward history to retrieve the users' information. For instance, if a user entered a query "last correspondence number", then the Chatbot parses the query into tokens such as "last", "correspondence", and "number". The parsed tokens will be passed in prioritized order and fetch the responses for the user query. The further development of the Chabot application will provide a complete form of this proposed application. In this initial phase, it presents users' last correspondence details according to their username.

\section{B. Sentiment Analysis}

The supervised learning approach is followed in the research for developing the proposed Sentiment Analysis technique. To implement the sentiment analysis using metadata, Python 3.8.1, and Power BI are employed. The primary sources for this proposed method are correspondences (Internal, Incoming, and Outgoing) and correspondence history. Power $\mathrm{BI}$ is used to generate the bag of words from the subject of the correspondences for each department. Fig. 6 shows one of the bags of words generated for a department. The token that has the highest frequency is shown in elevated form rather than other tokens.

NB algorithm is applied for the identification of the type of the correspondences. The identification process supports promoting the correspondence in order to complete it in a shorter amount of duration. The development process of sentiment analysis is in progress, and the current form differentiates the sent and received correspondences with its level. For instance, if a user creates a correspondence, then the proposed system automatically predicts the type of correspondence which supports administration to complete it within a limited amount of time. 
Hello! Mr. Test user, I'm a bot, Do you want to continue

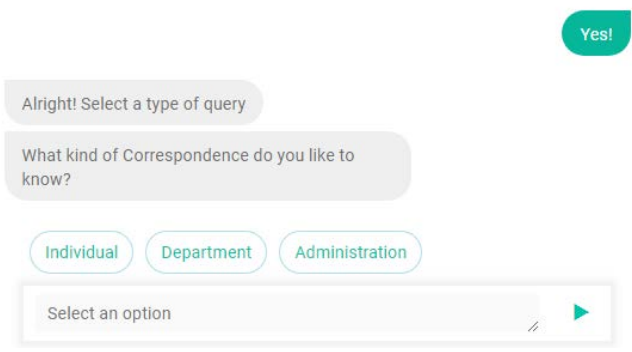

Fig. 5. Proposed Chatbot Application.

Bag of Words - Analyzer

token 10

token5 token8

token15 token3

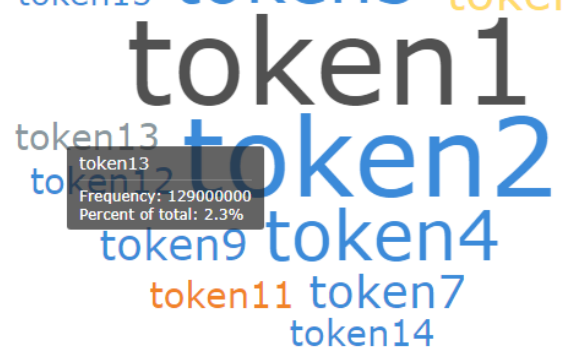

Fig. 6. Bag of Words.

\section{Key Performance Indicators}

A set of common KPIs for Root, Department, and Employee levels are identified via this study. The following is the list of KPIs proposed for government organizations: Root Level: Ranking workload, Estimating achievement percentage, Ranking Workload based on time, and Ranking Workload based on the number of forwards. Department Level: Estimating achievement time, Estimating achievement percentage, Actions per hour, Percentage of workload allocation, and Percentage of workload distribution. Employee Level: Estimating achievement time, Percentage of workload allocation, Estimating achievement percentage, and Actions per hour.

Oracle $11 \mathrm{G}$ in Windows 10 environment is employed to test the KPI and generated the results, and rendered the graphs using Canvas.Js, which provides Javascript API to render the graph. A KPI dashboard is developed to show the outcome of the KPIs as shown in Fig. 7 to 10. Fig. 7 presents the outcome of the KPI for the department level. It shows the performance of a specific department in an organization.

Fig. 7 and 8 illustrates the graph rendered via the proposed KPI for the department level. It supports the decision-maker to know that the achievement percentage and time of a department. Thus, the decision-maker will decide to enhance the performance of both departments and employees to complete the correspondences in time to improve the achievement percentage and time.
Fig. 9 shows the KPI insight for the employee level. For context, an employee received a sum of 1416 correspondences in the year 1440. The performance of that employee, action per hour is shown on the right side of Fig. 9. Fig. 10 shows the percentage of achievement for the employee. The employee has taken an average of 1.33 days to complete a correspondence that may affect the organization's overall performance.
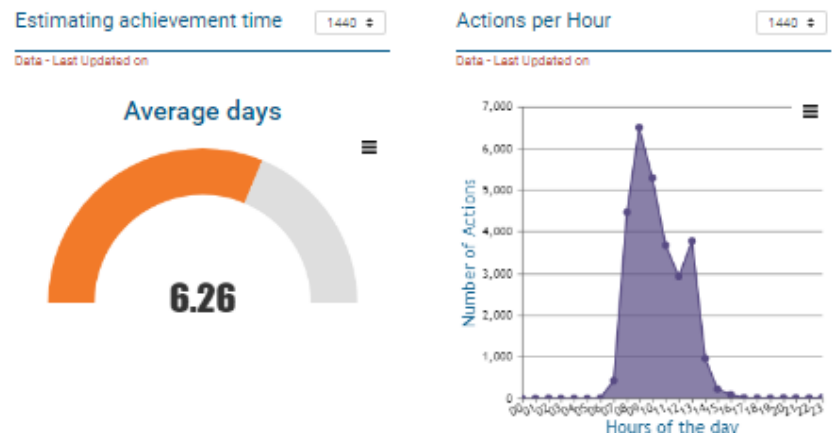

Fig. 7. KPI - Department Level -1 .

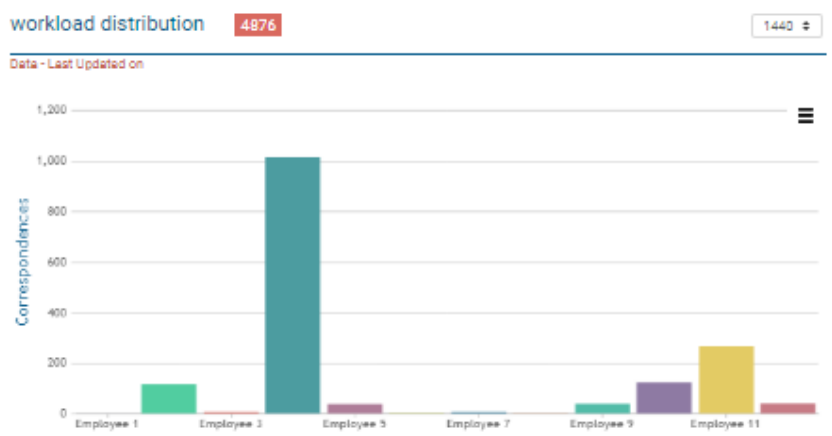

Fig. 8. KPI - Department Level -2 .

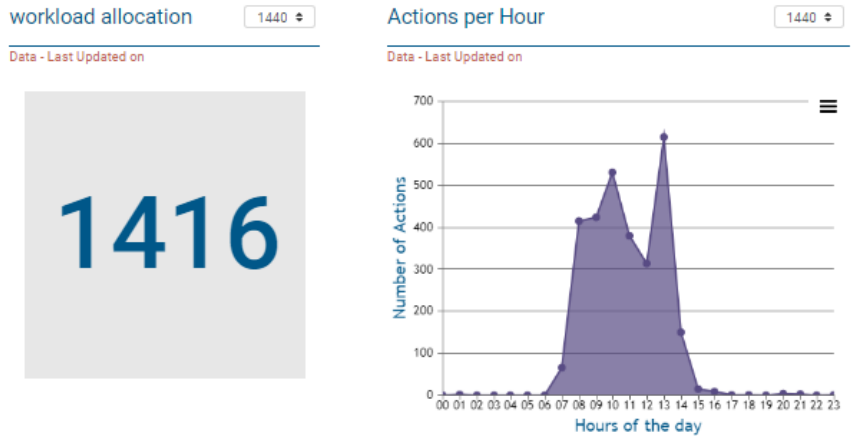

Fig. 9. Employee Level - 1.

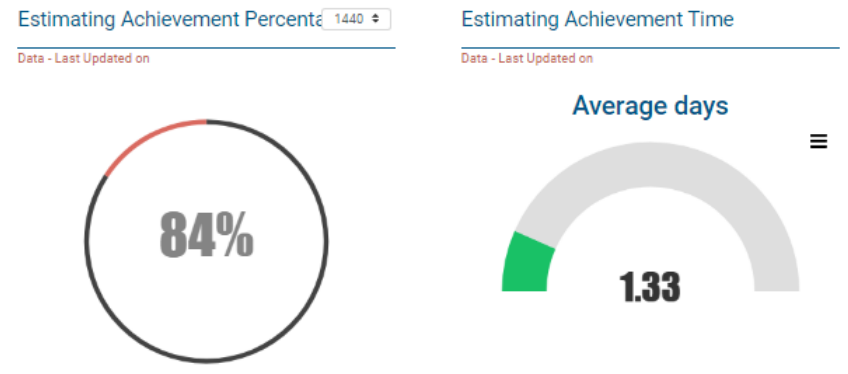

Fig. 10. Employee Level - 2. 


\section{Discussions}

The outcome of this study shows that metadata of a correspondence/document produces a sufficient amount of information for decision-makers to make an effective decision. More and more data linked to documents, the more alternatives are open for extracting observations, facts, and figures that assist in monitoring and tracking the workforce and complete a task in time. Such kind of data is a strong foundation and an essential element for the launch and implementation of highquality indicators. The National Centre for Archives and Records in Saudi Arabia has published a range of laws and policies on all kinds of documents that would strengthen administrative work and transactions. It includes an adequate database to store data concerning statistics and operational management indicators.

The results of this research show that the proposed framework can present an effective environment for an organization to manage its workforce. Both Chatbot and Sentiment Analysis support the organizations to respond to their clients' query automatically. Moreover, the KPI dashboard assists decision-makers in planning without any difficulties.

Operational and administrative processes share several characteristics [23]. Both involve sequences of linked, interdependent activities that together transform inputs into outputs. Both have clients who may be internal or external to the organization - the primary differences between the two lies in their outputs [24]. Typically, operational processes produce goods and services that external customers consume, while administrative procedures generate information and action plans for the internal groups [8]. For this reason, the two are frequently considered independent, unrelated activities, even though they must usually be aligned and mutually supportive if the organization is to function effectively.

The viewpoint of the work processes has contributed to a range of significant perspectives for executives [24]. It offers a beneficial context for a common organizational issue to be addressed: fragmentation or lack of integration. Many aspects of modern organizations, including complexity, highly segregated sub-units, and responsibilities, weak informal relationships, scale, and physical distance, make integration difficult [24]. The simple identification of work processes as viable units of study and goals of managerial action also enhances integration. For illustration, charting hierarchical workflow or pursuing an order through the fulfilment method are convenient ways to inform employees that the operations of various departments and regional units are interdependent, even though organizational charts indicate that beyond their vertical lines of authority [10].

Moreover, the viewpoint of the work processes offers fresh objectives for change. Managers discuss the fundamental mechanisms rather than concentrating on systems and functions. An apparent benefit is that they can analyze the organization's actual work closely.

Most of the improvement programs concentrated primarily on the restructuring of systems; the continued implementation and maintenance of the reconfigured systems were largely ignored [9][25]. However, without enough monitoring, coordination, and control, as well as periodic interference, even the best processes will not perform effectively. Furthermore, organizational processes were typically aimed at enhancement, although their enabling administrative functions were ignored [8]. Incompatibilities and contradictions occurred because there was no knowledge and preparations required for an efficient operation. The implementation of the Chatbot and Sentiment Analysis technique will support an organization to minimize the resources and satisfy the user requirements. The combination of hierarchical and traditional KPI can offer a better outcome to the organization to improve their performance.

The principles of information security support organizations to ensure unauthorized access to records [28]. For the proposed framework, some security concepts are framed as follows:

- Documents produced and accepted using this standard should be inspected for modifications, updates, or retirement of documents at regular intervals [29]. During regular document maintenance, the existing document owners must be verified or affirmed. However, it does not exceed three years from the previous revision date when the document is preserved ordinarily [30].

- The document developer must identify the process owners, service owners, and stakeholders and analyze the document during the development / initial analysis [30]. Although there is no prerequisite for any person or community review, it is highly recommended that members of those most affected by the content of the document should take part in the study.

- DMS must allow administrators to establish system permissions that are regulated based on the individual level of the document, ensuring that only approved staff has access to system files and documents [31]. Thus, confidential data is not in the hands of the wrong person, and the possibility of data breaches is greatly reduced.

As per the outcome of this study, the proposed Chatbot supports users to understand the status of their correspondence. It uses an AI approach to read the user query and provide a necessary response. The focus of the Chatbot is to minimize manual interactions with users. It works similar to an automated Chatbot in a banking system that responds to user queries. In the proposed Chatbot, the user needs to select an option to retrieve a response from BD. The proposed method uses a customized dataset depends on the employee and department. For context, an employee of level 2 requires his/ her correspondence information; the Chatbot searches his / her query in the level 2 dataset, not in the entire dataset. In case, data is not found in level 2; then the query will be matched with its higher level, i.e. level 1.

The proposed Sentiment analysis technique is able to predict the type of correspondence and support the decisionmaker / administrator to complete the transaction in a limited amount of time. In the existing model, the user needs to 
provide the type of correspondence. There is a possibility of error in this model. For instance, for an urgent task, the user may select a standard type instead of a critical type that leads the organization to postpone the urgent task to a later time. The proposed framework is capable of predicting the nature of correspondence automatically and assist the organization in increasing the achievement rate and turnaround time. The further development of this framework will lead to finding solutions for more challenges in a complex work environment.

The proposed research recommends implementing benchmark data derived according to the flow of documents to recognize best practices and growth opportunities. It is necessary to hold frequent conferences to enhance and exchange information related to archiving documents that will improve the role of IT in the archive service to become an intelligent centre and support decision-making processes. Moreover, developing a universal standard for archiving center supports administrative experts to construct, analyze, and plan for successful indicators. Before archiving a document and related data, a review must be performed to ensure the data supports the objectives and vision of the organization. Developing personalized and specialized courses can lead to a growth of professional human resources dealing with electronic documents and transactions.

Policymakers have an interest in AI. The three broader areas in which policymakers seek to ensure that organizations strike an adequate balance between their business interests and customers' interests includes: data protection, prejudice, and ethics [11][13][27]. Decision-makers need insights into how the data are collected to better understand and resolve privacy issues at present and how to handle failures in data privacy, especially with rules and regulations of National Archive and Records Centre, Kingdom of Saudi Arabia and Information security principles. Many AI algorithms are opaque black boxes, exacerbating the problem, and therefore it is hard to isolate the exact factors considered by these algorithms [25]. AI Organizations and developers must contend with ethics. Privacy decisions may represent the policy of an organization and could also be motivated by ethical concerns. In this context, research should look at "how regulatory ethics can pave the way for organizations to exceed consumer privacy expectations and oversubscribe to legal mandates to preserve self-regulation" [4]. A similar focus of research may be to explore how ethical questions regarding AI differ across cultures.

Finally, the proposed research indicates employees in the document centre to apply restrictions for protecting data during transactions. Data protection is one of the main steps towards ensuring the sustainability of the data. The protection processes should be initiated along with the creation of a correspondence. Data entered by users should be reviewed in order to maintain the correct format. It is also necessary to review the correspondence data to ensure that it supports the vision and mission of the organization to reach an optimal solution in the processes of data protection.

\section{CONCLUSION}

The kingdom's vision of 2030 has initiated many development programs in several areas and measure the same.
In the recent Global Summit AI, 2020, the new strategy for Data and AI is announced for implementing $\mathrm{AI}$ in the government and private sectors. The emergence of $\mathrm{BD}$ and $\mathrm{AI}$ techniques support organizations to identify the pitfalls that affect the performance and find a solution for improvement. The documents and their flows provide the documents and archives centre with an opportunity to make effective decisionmaking. Data granularity can feed information into a range of future activities and events. Since an organization is focused on predicting how risk is realized, having access to BD can change the entire working model of an enterprise/organization. However, the granularity of data may also contribute to the promotion of risk classification, where achievement rates are focused on a group of individuals with similar profiles. One of the statistical methods for representing information about an individual entity of an organization is the key performance indicator. An employee's success will significantly increase the performance of the relevant department. Therefore, it is necessary to evaluate individual performance and how it is beneficial to enhance an organization's overall performance. In this study, an AI-based Chatbot and Sentiment analysis were developed to support users to follow-up their correspondences. It has identified some useful key performance indicators that can measure the performance of employees at different levels. The initial results from Chatbot and Sentiment analysis are promising, and further development will provide a complete dimension to these applications. Also, the study has formulated and discussed the information security and AI policies to develop and maintain a document in the document centre.

REFERENCES

[1] Saudi Vision 2030, “www.vision2030.gov.sa,” 2017.

[2] E. Nica, Miklencicova Renata, and Kicova Eva, “Artificial intelligencesupported workplace decisions: big data algorithmic analytics, sensory and tracking technologies, and metabolism monitors," Psychosociological Issues Human. Resource Management, vol. 7, no. 2, pp. 31-36, 2019.

[3] Y. Duan, J. S. Edwards, and Y. K. Dwivedi, "Artificial intelligence for decision making in the era of big data - evolution, challenges and research agenda," International Journal of Information Management., vol. 48, pp. 63-71, Oct. 2019, doi: 10.1016/j.ijinfomgt.2019.01.021.

[4] K. D. Martin and P. E. Murphy, "The role of data privacy in marketing," Springer, no. 45, pp. 135-155, 2017, doi: 10.1007/s11747-016-0495-4.

[5] P. Patnaik, S. Pattnaik, and P. Singh, "Use of data analytics for effective E-Governance: a case study of 'EMutation' system of odisha," in Lecture Notes on Data Engineering and Communications Technologies, vol. 37, Springer, 2020, pp. 109-122.

[6] D. A. Bishop, "Key performance indicators: ideation to creation," IEEE Engineering Management, Rev., vol. 46, no. 1, pp. 13-15, Mar. 2018, doi: 10.1109/EMR.2018.2810104.

[7] M. Badawy, A. A. A. El-Aziz, A. M. Idress, H. Hefny, and S. Hossam, "A survey on exploring key performance indicators," Future Computing Informatics Journal., vol. 1, no. 1-2, pp. 47-52, Dec. 2016, doi: 10.1016/j.fcij.2016.04.001.

[8] J. H. K. Lai and C. S. Man, "Performance indicators for facilities operation and maintenance (Part 2): Shortlisting through a focus group study," Facilities, vol. 36, no. 9-10, pp. 495-509, Jul. 2018, doi: 10.1108/F-08-2017-0076.

[9] T. Dewett and G. R. Jones, "The role of information technology in the organization: a review, model, and assessment,” Journal Management, vol. 27, no. 3, pp. 313-346, Jun. 2001, doi: $10.1177 / 014920630102700306$.

[10] N. K. Dev, R. Shankar, R. Gupta, and J. Dong, "Multi-criteria evaluation of real-time key performance indicators of supply chain with consideration of big data architecture," Computing Industrial 
Engineering, vol. 128, pp. 1076-1087, Feb. 2019, doi: 10.1016/j.cie.2018.04.012.

[11] T. Davenport, A. Guha, D. Grewal, and T. Bressgott, "How artificial intelligence will change the future of marketing," Journal Academy Marketing Sciences, vol. 48, no. 1, pp. 24-42, Jan. 2020, doi: 10.1007/s11747-019-00696-0.

[12] D. Valle-Cruz, R. Sandoval-Almazan, E. A. Ruvalcaba-Gomez, and J. Ignacio Criado, "A review of artificial intelligence in government and its potential from a public policy perspective,” in ACM International Conference Proceeding Series, Jun. 2019, pp. 91-99, doi: 10.1145/3325112.3325242.

[13] T. Davenport, "Enterprise analytics: Optimize performance, process, and decisions through big data," 2013.

[14] C. Moreno, R. A. Carrasco, and E. Herrera-Viedma, "Data and Artificial Intelligence Strategy: A Conceptual Enterprise Big Data Cloud Architecture to Enable Market-Oriented Organisations," International Journal of Interactive Multimedia and Artificial Intelligence, vol. 5, no. 6, p. 7, Jun. 2019, doi: 10.9781/ijimai.2019.06.003.

[15] D. A. McFarland and H. R. McFarland, "Big data and the danger of being precisely inaccurate,” Big Data Society, vol. 2, no. 2, 2015, doi: $10.1177 / 2053951715602495$.

[16] M. Kowalczyk, "Key performance indicators in local government in Poland," Pr. Nauk. Uniw. Ekon. we Wrocławiu, no. 503, pp. 236-245, 2018, doi: 10.15611/pn.2018.503.21.

[17] A. Hooda and M. L. Singla, "Reengineering as a strategic stance for egovernance success - mediating role of core competencies: a mixed method study,” Transform. Gov. People, Process Policy, vol. 14, no. 2, pp. 205-235, May 2020, doi: 10.1108/TG-01-2020-0017.

[18] M. Y. Febrianta and H. Amani, "Identification of e-Governance indicators for measuring smart governance in bandung city,” 2019, doi: 10.31227/osf.io/avbsu.

[19] X. Luo, S. Tong, Z. Fang, and Z. Qu, "Frontiers: Machines vs. humans: The impact of artificial intelligence chatbot disclosure on customer purchases,” Mark. Sci., vol. 38, no. 6, pp. 937-947, Sep. 2019, doi: 10.1287/mksc.2019.1192.

[20] E. Pantano and G. Pizzi, "Forecasting artificial intelligence on online customer assistance: Evidence from chatbot patents analysis," Journal of Retail Consumers Serv., vol. 55, p. 102096, Jul. 2020, doi: 10.1016/j.jretconser.2020.102096.
[21] D. Agostino and M. Arnaboldi, "Rational and ritualistic use of key performance indicators in hybrid organizations," Public Money Management, vol. 37, no. 6, pp. 409-416, Sep. 2017, doi: 10.1080/09540962.2017.1344021.

[22] P. C. Verhoef et al., "Consumer Connectivity in a Complex, Technology-enabled, and Mobile-oriented World with Smart Products," Journal of Interactive Marketing, vol. 40, pp. 1-8, Nov. 2017, doi: 10.1016/j.intmar.2017.06.001.

[23] N. Gonzalez et al., "A quantitative analysis of current security concerns and solutions for cloud computing," Journal of Cloud Computing, vol. 1, no. 1, pp. 1-18, Jul. 2012, doi: 10.1186/2192-113X-1-11.

[24] E. Girdzijauskaitè, A. Radzevičienè, and A. Jakubavičius, "Impact of international branch campus KPIs on the university competitiveness: FARE method,” Insights into Reg. Dev., vol. 1, no. 2, pp. 171-180, Jun. 2019, doi: 10.9770/ird.2019.1.2(7).

[25] S. Mukherjee, "How IT allows E-participation in policy-making process,” arXiv:1903.00831, Mar. 2019, doi: 10.6084/m9.figshare.7796063.v2.

[26] A. Agrawal, J. Gans, and A. Goldfarb, "Economic policy for artificial intelligence,” Innov. Policy Econ., vol. 19, no. 1, pp. 139-159, Oct. 2019, doi: 10.1086/699935.

[27] J. Jung, R. Shroff, A. Feller, and S. Goel, “Algorithmic decision making in the presence of unmeasured confounding," arXiv Prepr. arXiv1805.01868, May 2018, Accessed: Sep. 21, 2020. [Online]. Available: http://arxiv.org/abs/1805.01868.

[28] S. Choi, J. T. Martins, and I. Bernik, "Information security: listening to the perspective of organisational insiders," J. Inf. Sci., vol. 44, no. 6, pp. 752-767, Dec. 2018, doi: 10.1177/0165551517748288.

[29] J. Roberts, “Organizational ignorance: towards a managerial perspective on the unknown,” Management Learning, vol. 44, no. 3, pp. 215-236, Jul. 2013, doi: 10.1177/1350507612443208.

[30] Z. Hussain, A. Taylor, and D. Flynn, "A case study of the process of achieving legitimation in information systems development," Journal Inf. Sci., vol. 30, no. 5, pp. 408-417, 2004, doi: $10.1177 / 0165551504046725$.

[31] L. Rasmussen and H. Hall, "The adoption process in management innovation: a knowledge management case study,” J. Inf. Sci., vol. 42, no. 3, pp. 356-368, 2015, doi: 10.1177/0165551515625032. 